\title{
"Stand-Out": A Novel Approach for Preparing Sub-100 nm Samples Through in situ Ion Induced Bending
}

\author{
Vivek Garg ${ }^{1,2,3}$, Shuo Zhang ${ }^{3}$, Rakesh G. Mote $^{2}$, Yu Chen ${ }^{4}$, Lingfei Cao ${ }^{5}$ and Jing Fu ${ }^{3}$
}

1. IITB-Monash Research Academy, Indian Institute of Technology Bombay, Mumbai, India.

2. Department of Mechanical Engineering, Indian Institute of Technology Bombay, Mumbai, India.

3. Department of Mechanical and Aerospace Engineering, Monash University, Clayton, Australia.

4. Monash Centre for Electron Microscopy, Monash University, Clayton, Australia.

${ }^{5}$ International Joint Laboratory for Light Alloys (Ministry of Education), College of Materials Science and Engineering, Chongqing University, Chongqing, China.

High resolution imaging techniques, such as Transmission electron microscopy (TEM) and Atom Probe Tomography (APT), are powerful tools for both materials and biological science. The success of imaging, however, rely heavily on sample preparation methods posing several challenges. Samples prepared for TEM need to be electron transparent $(50-100 \mathrm{~nm})$. Focused ion beam (FIB) has been a popular choice for site-specific TEM sample from bulk specimens and in-situ lift-out using a micromanipulator [1], [2][3]. This method, however, is time consuming and requires significant efforts and operator skills.

In this work, we introduce "stand-out", a novel sample preparation method capitalizing in-situ ion induced controlled bending for TEM applications. Gallium FIB is a dual beam FEI Quanta 3D system was used for fabrication of suspended nanowires (NWs), which were subsequently manipulated controllably through ion irradiation induced bending [4] to stand-out until becoming vertical to the sample surface. The method is shown step-by-step through schematics and scanning electron microscopic (SEM) images (Fig. 1). Trapezoidal trenches on the planar surface are milled to obtain a thin specimen wall, which is further used to obtain the suspended NW (100-200 nm) employing FIB under-milling (Fig. 1a). This suspended NW is cut at one end to enable the bending through controlled dose ion irradiation at the other end (Fig. 1b). The NW gradually aligns towards incident ion beam with increasing ion dose. The implanted-ions, generation of point defects, and dislocated lattice atoms contributes to the local development of stresses and ion irradiation induced plastic deformation. Further, annular milling is employed to thin the NW to enable the electron transparency required for TEM imaging (Fig. 1c). The capability of the proposed methodology is demonstrated through TEM sample preparation for different materials such as Silicon (Si), Tungsten (W), Molybdenum (Mo). A TEM image of a prepared Mo sample is presented (Fig. 2). Additionally, the developed stand-out method has the potential for atom probe tomography (APT) sample preparation in the form of a sharp needle with an end radius 50-100 $\mathrm{nm}$ without requiring the lift-out (Fig. 2). Thus providing a unique route for correlative imaging with TEM and APT yielding structural and chemical composition not possible previously [4]. In summary, the successful demonstration of stand-out, a novel sample preparation method encompassing different materials through in-situ ion induced bending in a dual beam FIB-SEM microscope provides a novel approach with a huge potential for TEM/APT applications even in a cryogenic environment [5].

\section{References :}

[1] LA Giannuzzi and FA Stevie, Micron 30 (1999), p. 197. 
[2] DW Saxey et al., Ultramicroscopy 107 (2007), p. 756.

[3] V Garg et al., Weaving Nanostructures with Site-Specific Ion Induced Bidirectional Bending (under review).

[4] M Herbig et al., Ultramicroscopy 153 (2015), p. 32.

[5] The work is financially supported by IITB-Monash Research Academy, the Australian Research Council (DP180103955), IRCC (Seed grant: Spons/ME/I14079-1/2014), IIT Bombay, and Tata Consultancy Services (TCS) research scholarship program. The authors acknowledge the use of facilities within the Monash Centre for Electron Microscopy (MCEM), and Melbourne Centre for Nanofabrication (MCN), Victorian Node of the Australian National Fabrication Facility (ANFF).

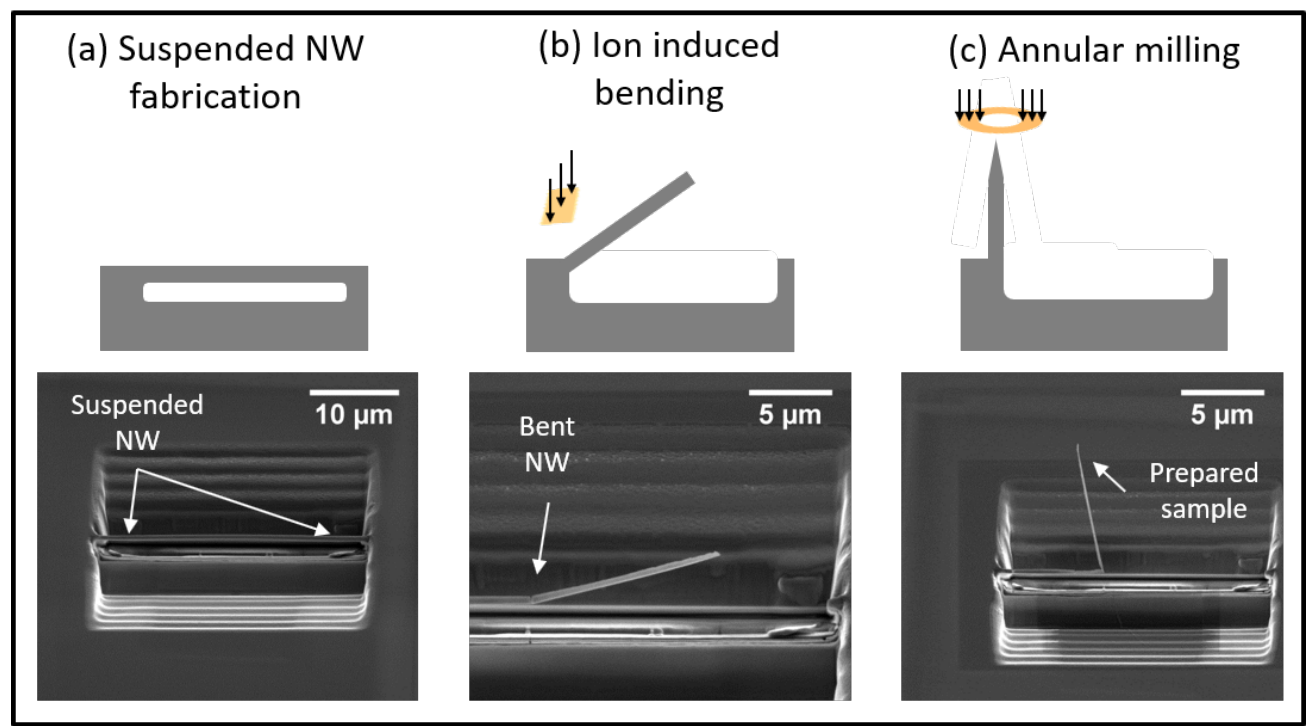

Figure 1. Step-by-step illustration of Stand-out sample preparation method for TEM/APT in a dual beam FIB-SEM microscope: schematic diagrams and SEM images showing (a) fabrication of suspended nanowire (NW), (b) ion induced bending for vertical alignment, (c) annular milling for final polishing

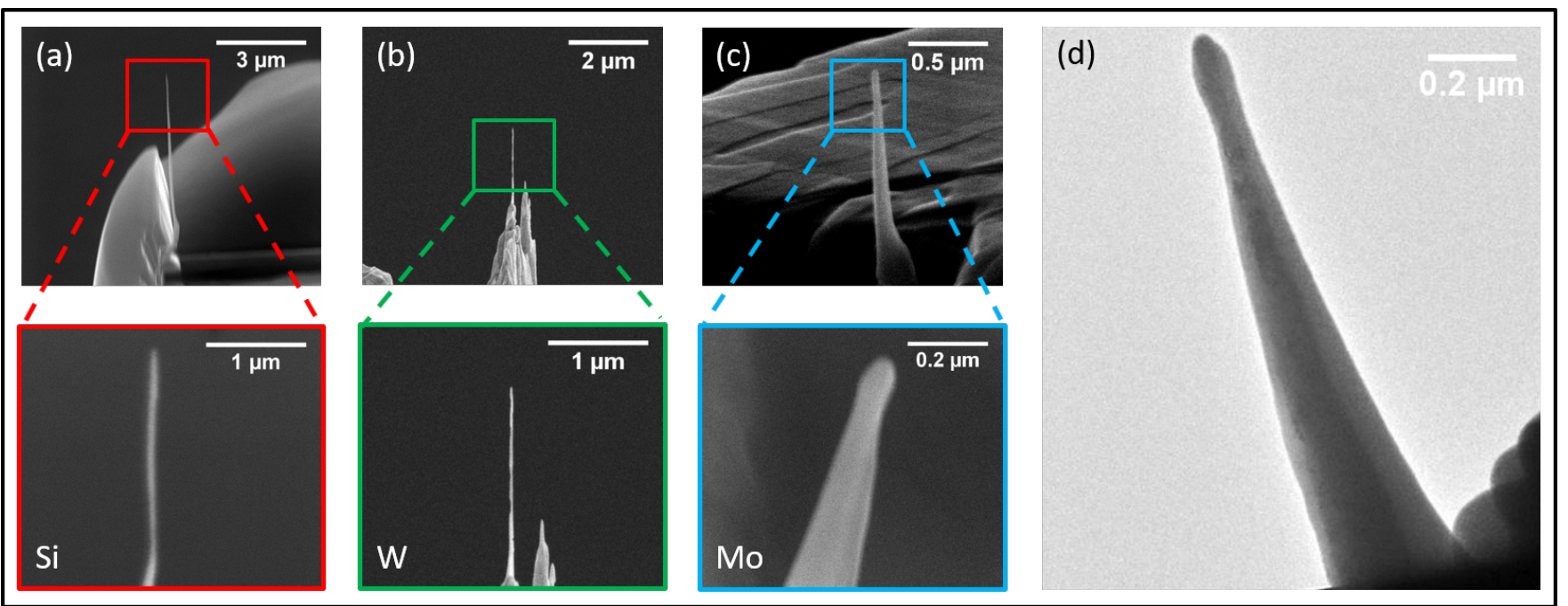

Figure 2. SEM images of samples prepared on different materials using stand-out method in a dual beam FIB-SEM microscope: sub $100 \mathrm{~nm}$ sample of (a) Si, (b) W, (c) Mo. (d) TEM image of a Mo sample prepared by the "stand-out" approach. 